\title{
Gene Therapy: Possibilities and Promise
}

\author{
LARRY J. SHAPIRO
}

Department of Pediatrics, University of California Medical Center, San Francisco, California 94143

It is now nearly 50 years since DNA was discovered to be the repository of hereditary information and approximately 20 years since the recombinant DNA era began. From the earliest times in this scientific saga, there have been hopes that it would be possible to transfer therapeutically effective genes into human patients just as one can do with bacteria or mammalian cells in culture. As our knowledge and technologic skills have increased, the likelihood of being able to accomplish these goals has risen. In this issue of Pediatric Research, a consortium of investigators describes some of the most recent work that they hope will lead to the establishment of protocols for transfer of exogenous genetic material into isolated hepatocytes that can then be infused into recipients. Although much work remains to be done, it is appropriate to take stock of how far scientists in this field have come and what the major issues are that are currently being confronted. If having a scientific journal devoted to a discipline (Human Gene Therapy) and receiving extensive coverage in the lay press are indicators of having come of age, then this area of endeavor certainly has arrived.

When thinking about gene therapy, most people attempt to discriminate between treatments designed to alter somatic cells and therapies that could alter the germline and thus be transmitted to future generations. There is general agreement that germline gene therapy would be inappropriate to contemplate at present given the unknown potential for long-term harm of foreign genes, particularly those with the possibility of being expressed in every cell of the body and throughout development (as in transgenic mice). Although there are those who oppose any efforts to use DNA as a therapeutic agent, it can be argued very forcefully that somatic cell gene therapy does not differ from other current treatment strategies in a fundamental or conceptual way. All new pharmacologic agents carry some potential for toxicity and risk that needs to be evaluated in a careful and systematic manner. Cancer chemotherapeutic agents, as one example, also possess the ability to mutagenize or alter the DNA of cells in the recipient and are certainly associated with substantial toxicity. However, DNA has a special cachet and admittedly is one of a few therapeutic agents being used that has the potential to replicate itself (as do live viral and bacterial vaccines). It would appear that the novelty of gene therapy and the need to assuage the fears and concerns of a number of citizens has resulted in a regulatory process and a level of discussion that is unique. Perhaps sensitivities have been further heightened by a misadventure of the early 1980s in which a U.S. scientist began a clinical gene therapy experiment without appropriate approvals. In the United States, a separate panel, the NIH Recombinant DNA Advisory Committee, has been established to review and approve potential protocols in addition to the more traditional involvement of local Institutional Review Boards and the Food and Drug Administration. At present, there are more than 10 active clinical protocols, an additional 12 that have been approved but have not yet enrolled any patients, and more than a dozen other proposals in various stages of development.

A variety of goals exist for the new breed of gene therapists, and new possibilities are almost continuously envisioned. Treatment of genetic diseases with replacement of a defective gene's function is being explored in lymphocytes and stem cells of patients with adenosine deaminase deficiency, in hepatocytes of patients with LDL receptor defects, and in fibroblasts engineered to produce factor IX for hemophilia B patients. Cancer and infectious diseases are also targets for these new modalities. Tumor-infiltrating lymphocytes are being given genes that will cause them to overproduce cytokines and, by homing to tumors, to deliver them locally. Melanoma cells are being treated to induce them to produce foreign HLA antigens and thus become targets for immune rejection. Ovarian cancer cclls are being manipulated so that they produce herpes thymidine kinase and so become uniquely susceptible to the drug ganciclovir. Many other proposals are under consideration, such as the infection of suitable target cells with vectors that will overproduce the TAR region of the human immunodeficiency virus genome and therefore competitively bind the viral transactivator protein "tat" and interfere with viral replication.

The first gene transfer protocol to be approved was actually designed to mark infused tumor-infiltrating lymphocytes with a DNA tag that would not alter cellular physiology but would enable investigators to sensitively and specifically follow the fate of these cells when given to patients. Another example of such a use is to insert a marker gene into purged autologous marrow samples harvested in remission and used to rescue leukemia or neuroblastoma patients given potentially lethal chemotherapy and/or radiation treatment. this would allow the investigator to follow the fate of the transplanted cells to see how they repopulate the marrow and to ask in case of a recurrence of tumor whether this is due to residual tumor cells in the patient that had not been eradicated or is the result of occult malignant cells in the transplanted marrow. The ability to interpret the results of such experiments is highly dependent on the efficiency with which donor marrow cells can be marked by gene transfer. The studies outlined in the article by Ledley et al. in this issuc have a related rationale. They propose that hepatocellular transplantation (either autologous or heterologous) may be a clinically useful modality. Hepatocyte gene transfer is a useful adjunct in two ways: first, as a means to mark donor liver cells so that their fate can be easily assessed, and second, because it may be useful to try to get these transplanted hepatocytes to produce and secrete some desired protein. They have therefore undertaken a variety of preclinical studies in several different animal models.

Many technical problems remain to be solved for these investigators as well as others venturing into the new world of gene transfer. Optimal vector and delivery systems must be perfected. At the present time, most efforts use defective retroviral delivery systems. Although they are efficient at delivering their payload, they are relatively indiscriminant as to the cells they infect and their use does not result in integration of DNA into a consistent or regulated site in the recipient genome. They also have a fairly modest capacity as to the amount of DNA that they can contain. Furthermore, there are some safety considerations; recent experiments in monkeys have shown that the helper viruses required to propagate some of these defective vectors may be oncogenic. Extreme care will be needed to ensure that virus preparations are free of such contaminants. The random integration mentioned above is a problem for controlling the expression of the introduced genes and also theoretically in terms of safety inas- 
much as activation or disruption of certain resident cellular genes could potentially be harmful. Other types of vectors, some with possible specificity of cellular tropism, are also being developed. In addition to delivering DNA with the proper regulatory sequences to a permissive genomic location, the proper cell type must be infected both to accomplish the desired clinical effect and to achieve persistence by modulating the genome of a longlived or renewable cell population. Some investigators are also concerned that an ex vivo approach of gene therapy requiring cell culture will be costly and inefficient and will not be applicable to all situations. Therefore, methods of DNA transfer using either naked DNA or liposomes by direct injection (into muscle), particle acceleration (into skin and other organs), and inhalation are all receiving considerable attention. There is still much to be learned about how to introduce genes into the proper cells with high efficiency and how to achieve carefully regulated levels of gene expression.

Hepatocellular gene therapy and transplantation are particularly attractive areas of investigation. Many disease states might be amenable to such interventions. However, these newer methods will have to compete with more established treatments such as liver transplantation and, more recently, living related donor hepatic transplants and reduced size organ grafts, which help to overcome some of the problems of organ availability. Xenografts are another potential solution for this issue. It is unclear whether heterologous hepatocytes will be any more readily available than heterologous intact livers. Furthermore, the validity of the assumption of Ledley et al. that donor livers unsuitable for transplantation would be useful as a source of hepatocytes is unproven. Harvesting autologous hepatocytes from very sick patients for ex vivo manipulations may present significant clinical problems as well. In addition, transplantation of the liver involves more than just hepatocytes (e.g. Kupffer cells). Other types of cells may be needed to repair certain disease states. Finally, it is not clear what percentage of the normal liver parenchyma needs to be augmented by transplanted hepatocytes to obtain a desired therapeutic outcome. If the number of donor cells must be very large, hemodynamic and functional problems related to embolization of the portal circulation will need to be carefully evaluated.

We are at the very beginning of an exciting new era that has the potential to revolutionize the therapy of a number of human disorders. Many additional interesting opportunities will likely develop as the Human Genome Initiative progresses and new genes are identified. Many of the earliest advances are likely to specifically benefit pediatric patients, and the careful collaboration of a diverse array of basic scientists and clinical investigators will need to occur to ensure that new ideas are developed, refined, and evaluated in a responsible way. 